\title{
Energy equipartition and frequency distribution in complex attachments
}

N. Roveri, A. Carcaterra, and A. Akay

Citation: The Journal of the Acoustical Society of America 126, 122 (2009); doi: 10.1121/1.3147502

View online: http://dx.doi.org/10.1121/1.3147502

View Table of Contents: http://asa.scitation.org/toc/jas/126/1

Published by the Acoustical Society of America 


\title{
Energy equipartition and frequency distribution in complex attachments
}

\author{
N. Roveri \\ Department of Mechanics and Aeronautics, University of Rome, "La Sapienza," Via Eudossiana 18, 00184 \\ Rome, Italy
}

\begin{abstract}
A. Carcaterra ${ }^{\text {a) }}$
Department of Mechanics and Aeronautics, University of Rome, "La Sapienza," Via Eudossiana 18, 00184

Rome, Italy and Department of Mechanical Engineering, Carnegie Mellon University, Pittsburgh,

Pennsylvania 15213
\end{abstract}

\author{
A. Akay \\ Department of Mechanical Engineering, Bilkent University, 06800 Bilkent, Ankara, Turkey and Department \\ of Mechanical Engineering, Carnegie Mellon University, Pittsburgh, Pennsylvania 15213
}

(Received 13 November 2008; revised 26 April 2009; accepted 11 May 2009)

\begin{abstract}
As reported in several recent publications, an undamped simple oscillator with a complex attachment that consists of a set of undamped parallel resonators can exhibit unusual energy sharing properties. The conservative set of oscillators of the attachment can absorb nearly all the impulsive energy applied to the primary oscillator to which it is connected. The key factor in the ability of the attachment to absorb energy with near irreversibility correlates with the natural frequency distribution of the resonators within it. The reported results also show that a family of optimal frequency distributions can be determined on the basis of a variational approach, minimizing a certain functional related to the system response. The present paper establishes a link between these optimal frequency distributions and the energy equipartition principle: optimal frequency distributions are those that spread the injected energy as uniformly as possible over the degrees of freedom or over the modes of the system. Theoretical as well as numerical results presented support this point of view. (C) 2009 Acoustical Society of America. [DOI: 10.1121/1.3147502]
\end{abstract}

PACS number(s): 43.40.At, 43.40.Kd, 43.40.Jc, 43.40.Tm [JJM] Pages: 122-128

\section{INTRODUCTION}

In the past decade the problem of energy sharing among a principal, or master, structure attached to a large number of resonators has been analyzed in some detail (viz., Refs. 1-3). Mechanism of energy sharing in a complex structure as depicted by the prototypical system described here brings out important fundamental issues in such systems and also has numerous engineering applications. Many engineering structures follow a similar paradigm where a population of resonators is attached to a principal structure. For example, structures such as a car body, airplane fuselage, or hull of a ship are coupled to a very large number of resonating interior components. Moreover, the fundamental aspects of the concept of complex attachments can be used for designing novel vibration absorbers. ${ }^{4}$ The energy exchange that takes place in the complex system described here is substantially independent of any intrinsic damping in the system. ${ }^{1-8}$

Theoretical analyses that have been reported in a series of recent papers ${ }^{9-13}$ describe how a complex attachment can rapidly and permanently absorb energy from a master structure. One of the basic findings of these investigations was the discovery of the significance of the distribution of the natural frequencies of the attached resonators in this energy transfer

\footnotetext{
a) Author to whom correspondence should be addressed. Electronic mail: a.carcaterra@dma.ing.uniromal.it
}

process. The reported analyses revealed the existence of a family of special frequency distributions that can lead to trapping of the energy within the attachment, leading to a phenomenon called near-irreversibility. ${ }^{11}$ A common characteristic of this family of frequencies is the presence of a singularity or frequency concentration point in their distribution. ${ }^{11-13}$

A rather intriguing aspect of the energy-exchange phenomenon investigated here relates to the distribution of energy among the resonators and the frequencies of the oscillators. For most frequency distributions, the energy transferred to the attachment is largely confined to a limited number of resonators. ${ }^{8,9}$ In these cases, after an interval, the duration of which is theoretically predicted in Ref. 8, the resonators become in-phase with one another and the energy is suddenly returned to the master. However, this energy return effect is not observed for those special frequency distributions introduced in Refs. 9 and 12, energy remains within the set of oscillators and is spread over the resonators rather uniformly. ${ }^{9}$

The link between the optimal frequency distributions defined in Ref. 12 and flow of energy from the master to the attached resonators is the subject of the present paper. Of particular interest is the idea that the optimal frequency distributions are akin to a requirement of energy equipartition among the degrees of freedom of the system, or over its modes, which maximizes the trapped energy within the at- 


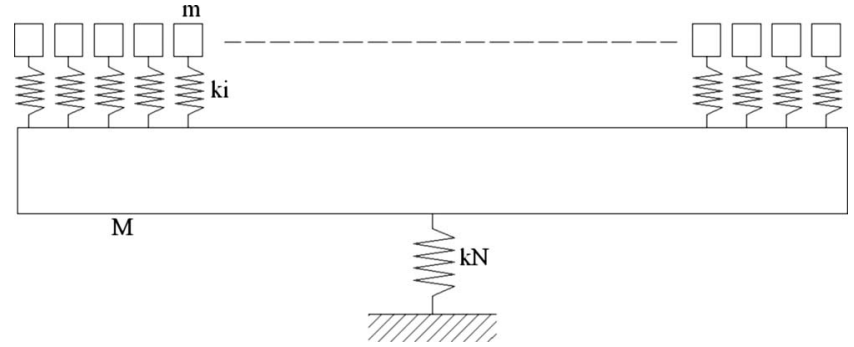

FIG. 1. Schematic of the master and the complex attachment.

tachment. When established, such a link can clarify how energy equipartition allows the master to keep only a small fraction of the total energy, thus having the attachment act as an effective vibration absorber.

The notion of energy equipartition in dynamics has deep roots and strong analogies in thermodynamics. For instance, in molecular mechanics, it is well known that the condition of thermalization, characterized by a uniform distribution of the energy among the molecules, is a condition to which physical systems approach as a consequence of the maximum entropy principle. Reaching thermalization implies that the system has gained its equilibrium and its macro-scale energy distribution has become stable. Based on this notion, this paper hypothesizes that if the oscillations of the attached resonators can reach a state of thermalization by a suitable selection of their natural frequencies, it can then be expected that the system will have a stable energy distribution. In other words, thermalization of the attached oscillators will avoid any periodic energy transfer between the master and the attached oscillators, de facto leading to an irreversible energy transfer as discussed in but following different criteria than those reported in Ref. 9 and in Refs. 11-13.

In Sec. II, the question of energy equipartition is considered using modal energies; in Sec. III, the problem is reexamined in terms of energy equipartition among the degrees of freedom of the system. As shown in Appendix, for the particular system investigated here, both forms of energy equipartition requirements are substantially the same.

\section{FREQUENCY DISTRIBUTIONS THAT LEAD TO MODAL ENERGY EQUIPARTITION}

The equations of motion of the system represented in Fig. 1 are

$$
\begin{aligned}
& m \ddot{x}_{j}+k_{j}\left(x_{j}-x_{N}\right)=0, \quad j=1,2, \ldots, N-1, \\
& M \ddot{x}_{N}+k_{N} x_{N}+\sum_{j=1}^{N-1} k_{j}\left(x_{N}-x_{j}\right)=0
\end{aligned}
$$

where index $N$ represents the master and $1,2, \ldots, N-1$ represent the oscillators of the attachment; $m$, and $k_{j}$ are the mass and the stiffness of each oscillator of the attachment, and $M$ and $k_{N}$ represent the mass and the stiffness of the master, respectively; $x_{j}$ is the displacement of the $j$-th oscillator. Expressing Eq. (1) in matrix form:

$$
\mathbf{M} \ddot{\mathbf{x}}+\mathbf{K x}=\mathbf{0},
$$

where $\mathbf{M}, \mathbf{K}$ are the mass the stiffness matrices. The use of modal coordinates $\boldsymbol{\eta}$ through the eigenvector matrix $\mathbf{U}$ produces

$$
\mathbf{x}=\mathbf{U} \boldsymbol{\eta} .
$$

Expressing the modal energies as

$$
E_{j}=\frac{1}{2}\left(\dot{\eta}_{j}^{2}+\omega_{j}^{2} \eta_{j}^{2}\right)
$$

where $\omega_{f}^{2}$ are the eigenvalues of the system, for an initial impulse $M V_{0}$ imparted to the master, Eq. (4) takes the following form:

$$
E_{j}=\frac{V_{0}^{2}}{2}\left(\Psi_{j N}\right)^{2},
$$

where $\boldsymbol{\Psi}=\mathbf{U}^{-1}$. Modal energies depend explicitly on the system eigenvectors, and indirectly on the set of physical parameters $m, k_{j}, M$, and $k_{N}$ of the system. $M$ and $k_{N}$ are given, as well as the total mass of the attachment $m(N-1)$, a small fraction of the master mass $M$. Therefore, $E_{j}$ varies with the values of $k_{j}(j=1,2, \ldots, N-1)$ or equivalently depends on the set of the uncoupled natural frequencies $\Omega_{j}=\sqrt{k_{j} / m}$ of the attached oscillators.

Modal energy equipartition where the total energy $E_{\text {tot }}$ spreads uniformly over the modes of the system can be expressed as

$$
E_{j}\left(\Omega_{1}, \ldots, \Omega_{N-1}\right)=E_{j}(\boldsymbol{\Omega})=\frac{E_{\text {tot }}}{N}, \quad j=1,2, \ldots, N .
$$

The frequencies $\Omega_{j}$ that lead to modal equipartition can be obtained by applying a least squares procedure to minimize the error function $\varepsilon$ :

$$
\varepsilon(\boldsymbol{\Omega})=\sum_{j=1}^{N}\left[E_{j}(\Omega)-\frac{E_{\text {tot }}}{N}\right]^{2} .
$$

The algorithm starts with an initial guess $\boldsymbol{\Omega}^{\text {in }}$ for the frequency distribution and stops when a specified convergence criterion is satisfied.

For the three different initial guesses shown in Fig. 2, the final distribution obtained through the minimization algorithm is the same for each, as shown in Fig. 3. Apparently, the results do not depend on the initial estimate for the frequency distribution. The optimal distribution is characterized by an inflection point in the neighborhood of the master frequency where its slope is close to zero. As a consequence, the modal density has a sharp peak around the master frequency, same as for those obtained in Ref. 12, but in this case using different optimization criteria.

In Fig. 4, the modal energy spectra corresponding to the three initial guess distributions are plotted, while the flat line represents the spectrum related to the optimal distribution that produces equipartition of the modal energies, determined through minimization of $\varepsilon$.

In Figs. 5 and 6, energy-time histories of the master are plotted for linear and optimal distributions, respectively (time is normalized with respect to the uncoupled natural 


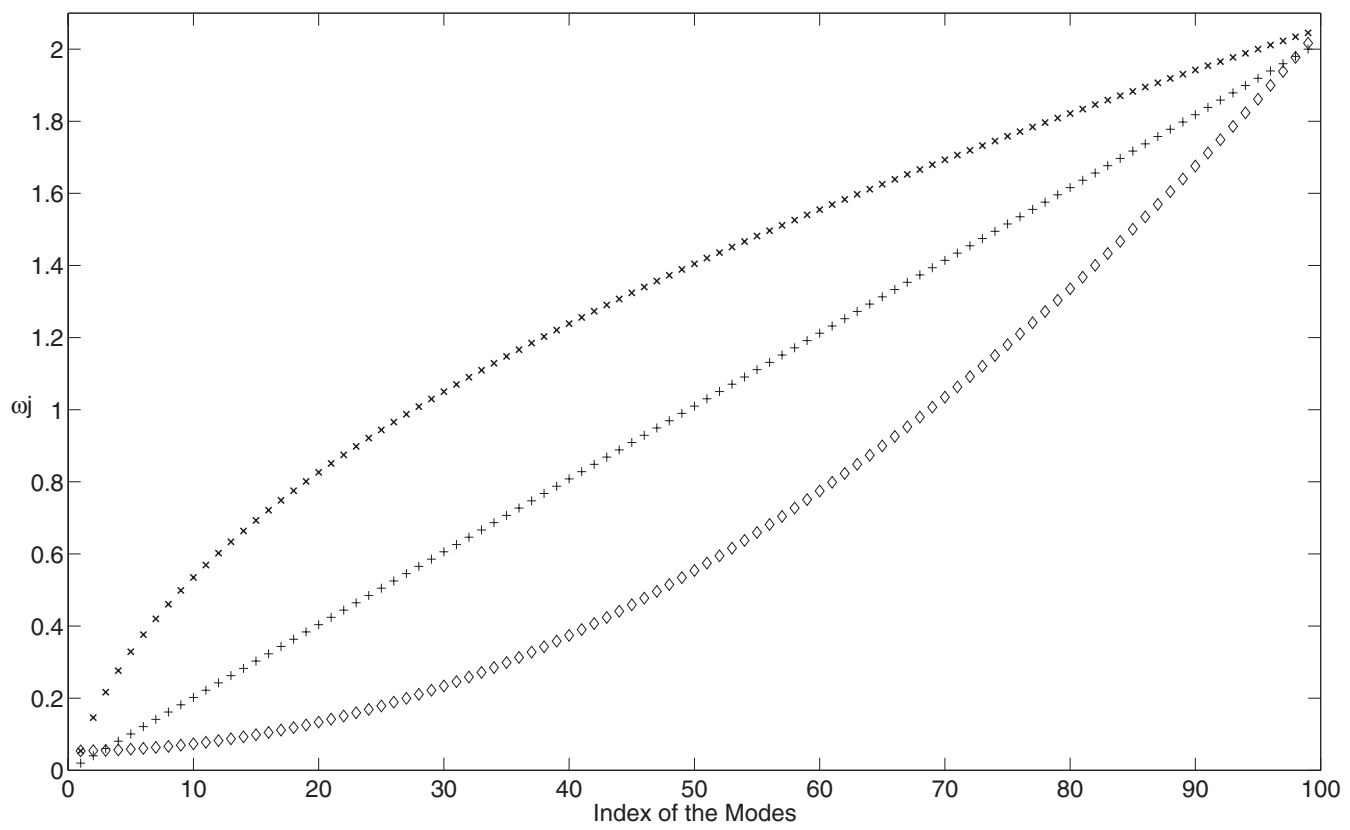

FIG. 2. Initial guesses for frequency distributions of the secondary structure with 99 degrees of freedom:,$+ \times$, and $\diamond$ are for the linear, exponential, and quadratic distributions, respectively.

frequency of the master). These figures demonstrate how an attachment with the optimal frequency distribution is able to minimize the energy stored in the master: after an initial transient, the secondary structure acts as an apparent damper and absorbs, almost completely, the total energy in the system.

\section{ENERGY EQUIPARTITION AMONG THE OSCILLATORS OF THE ATTACHMENT}

The theoretical developments in this section attempt to provide insight to the notion that frequency distributions leading to energy equipartition have particular forms. As shown in Sec. IV and further explained below, the main char- acteristic of these frequency distributions is the presence of a minimum slope around the master frequency that also corresponds to a large peak in the associated modal density.

The following theoretical analysis considers the requirement for energy equipartition among the oscillators of the attachment instead of among the modal energies of the system, which was considered in Sec. II. The connection between these two approaches will be discussed later in this section.

As shown in Ref. 12, Eq. (1) can be approximated by a continuous distribution of oscillators attached to the master, replacing the summation by an integral, and the index $i$ by a continuous variable $\xi$ :

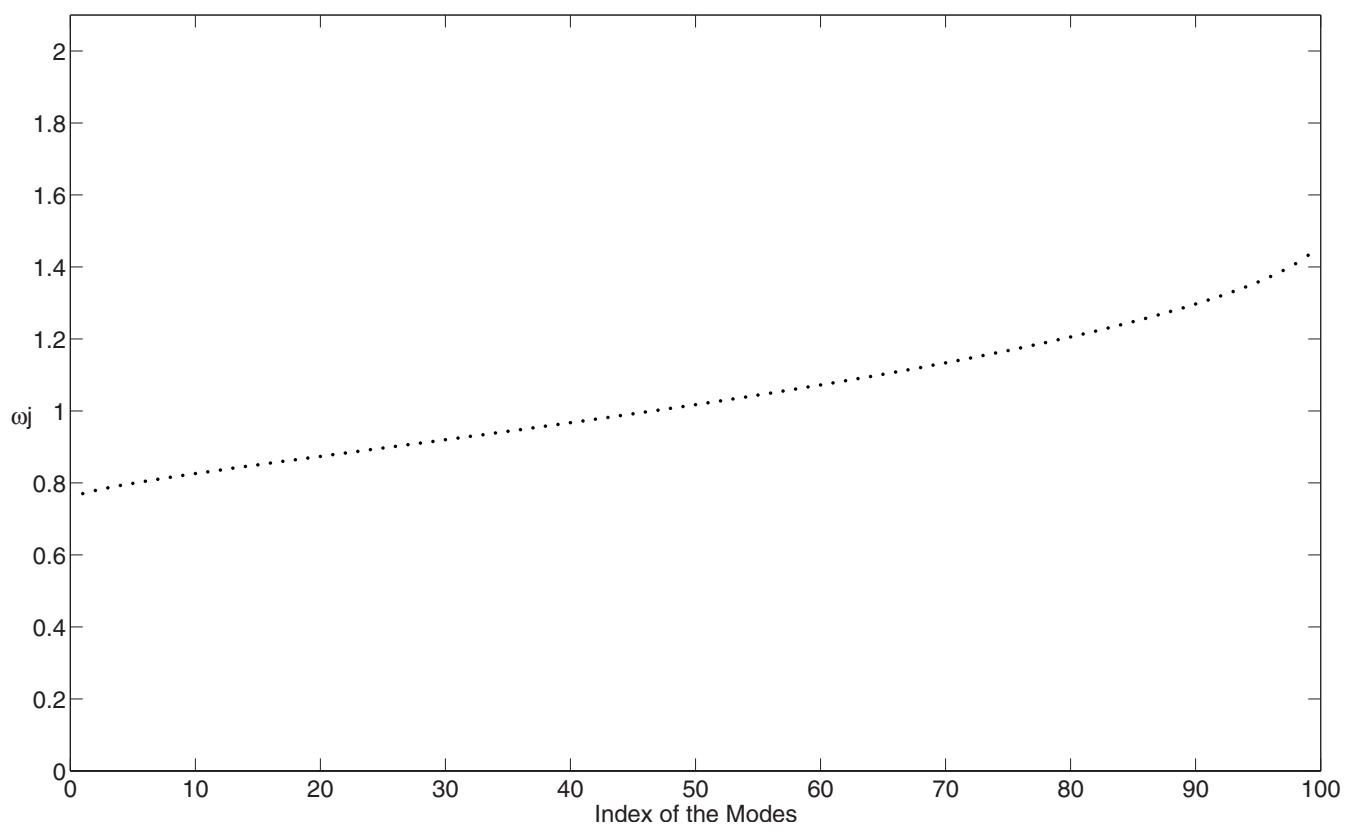

FIG. 3. Optimal frequency distribution in the attachment. 


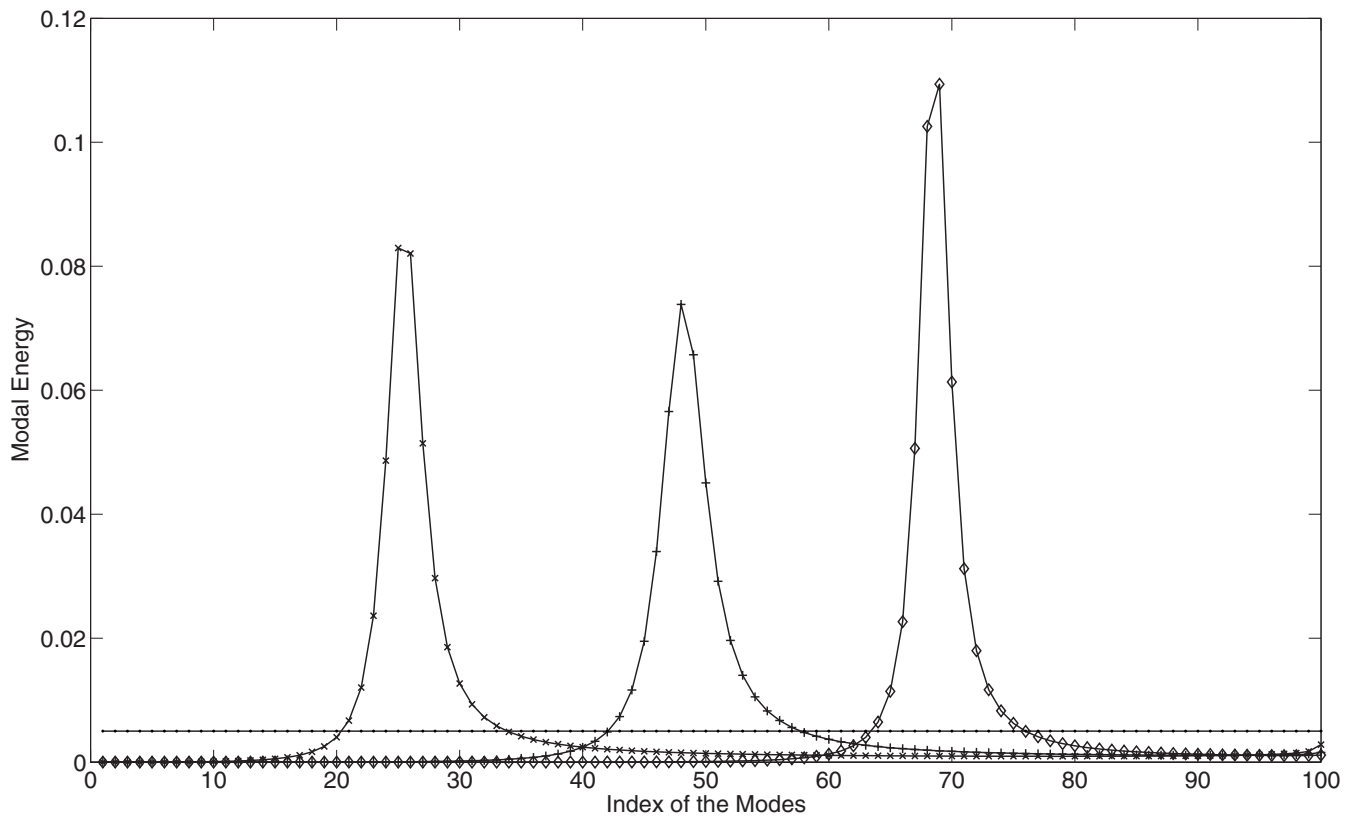

FIG. 4. Modal energy spectra related to the selected frequency distributions;,$+ \times$, and $\diamond$ are for the linear, exponential, and quadratic distributions, respectively.

$$
\begin{aligned}
& M \ddot{x}+k_{N} x+\int_{0}^{1} k(\xi)[x-x(\xi)] d \xi=f, \\
& m(\xi) \ddot{x}(\xi)-k(\xi)[x-x(\xi)]=0 .
\end{aligned}
$$

A detailed discussion about the meaning and the limits of this approximation is given in Refs. 8 and 12. Physically, such an approximation implies that the finite set of resonators is replaced by an infinite set, and thus as $N$ approaches infinity, the frequency gap between neighboring resonators vanishes. Expressing $x(\xi)$ in terms of $x$ in the second equation of Eq. (8), and substituting it into the first equation in Eq. (8), leads to an explicit expression for $x$, producing an approximate frequency domain counterpart of Eqs. (8), see Ref. 12:

$$
\begin{aligned}
& -M \omega^{2} X+k_{N} X+j \omega C_{\mathrm{eq}}(\omega) X=F, \\
& -\rho_{0} \omega^{2} X(\xi)+k(\xi)[X(\xi)-X]=0,
\end{aligned}
$$

where $F, X$, and $X(\xi)$ are the Fourier transforms of $f, x$, and $x(\xi)$, respectively, and a uniform mass distribution $m(\xi)=\rho_{0}$ is assumed. The equivalent damping is represented as

$$
C_{\mathrm{eq}}(\omega)=\left.\rho_{0} \frac{\pi}{4} \omega^{2} \frac{1}{d \omega_{n}(\xi) / d \xi}\right|_{\omega_{n}=\omega_{M}},
$$

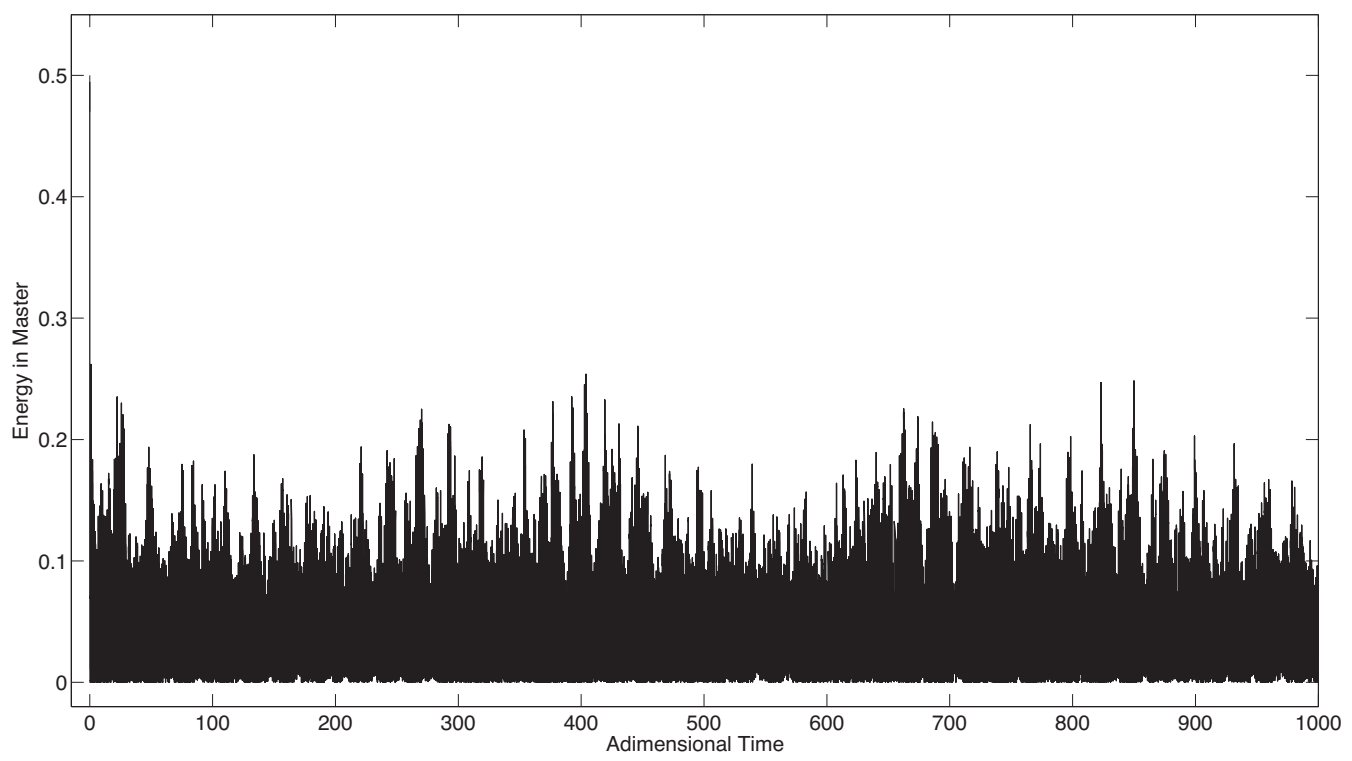

FIG. 5. Time history of the master energy plotted for linear distribution of the uncoupled frequencies of the satellite resonators, $N=100$; time is normalized with respect to the highest modal period: $T_{1}$. The non-dimensional energy of the complete system is 0.5 . 


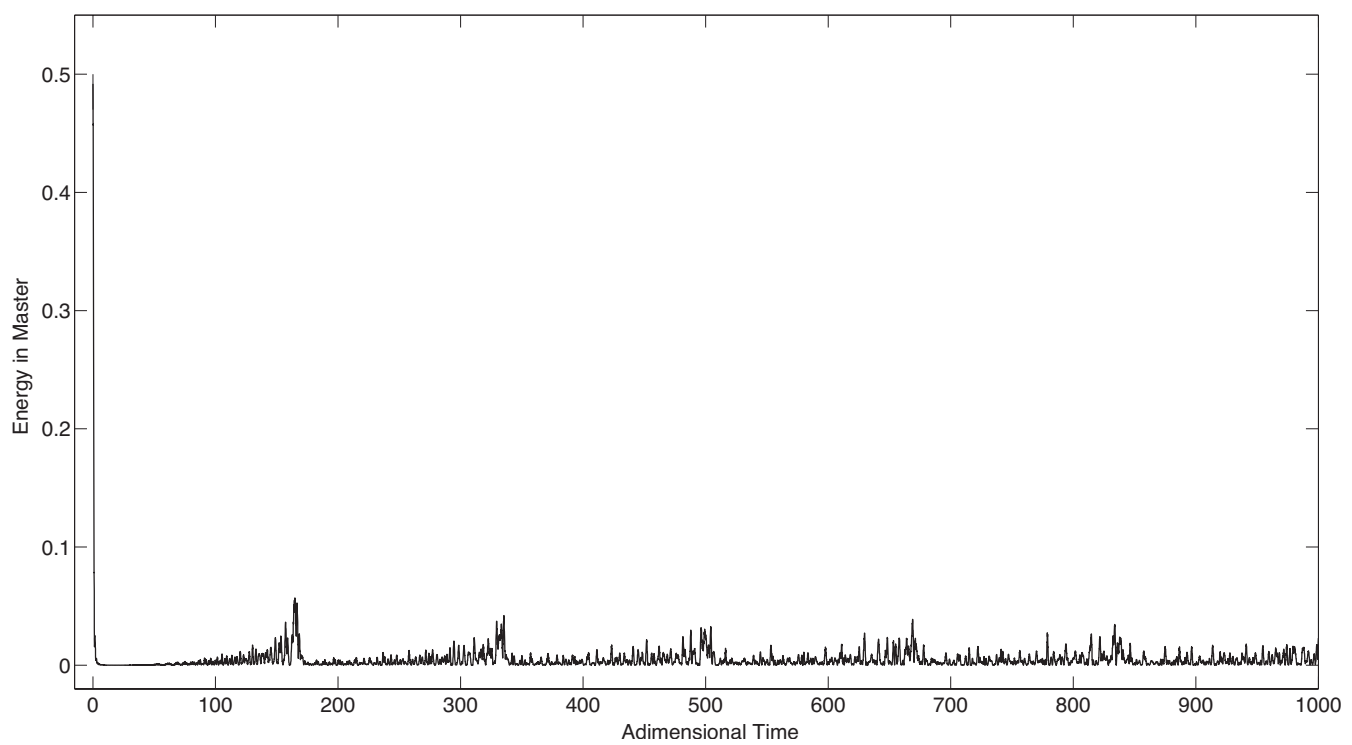

FIG. 6. Time history of the master energy plotted for optimal frequency distribution of the uncoupled frequencies of the satellite resonators, $N=100$.

$$
\text { with } \omega_{n}(\xi)=\sqrt{\frac{k(\xi)}{\rho_{0}}}
$$

as the natural frequency distribution within the attachment. Solutions to Eq. (9) are

$$
\begin{aligned}
& X=\frac{F}{-M \omega^{2}+k_{N}+j \omega C_{\mathrm{eq}}(\omega)}, \\
& X(\xi)=\frac{\omega_{n}^{2}(\xi)}{\omega_{n}^{2}(\xi)-\omega^{2}} \frac{F}{-M \omega^{2}+k_{N}+j \omega C_{\mathrm{eq}}(\omega)} .
\end{aligned}
$$

Expressing the impulsive force as $F=M V_{0}$, with $\omega_{M}$ $=\sqrt{k_{N}} / M$ and $\mu=\rho_{0} / M$, the total energy distribution $E(\xi)$ within the attachment is found by doubling the potential energy at any $\xi$ :

$$
\begin{aligned}
E(\xi) & =k(\xi)|X(\xi)-X|^{2} \\
& =\frac{1}{\omega_{n}^{2}} M V_{0}^{2} \mu \frac{\omega^{4} \omega_{n}^{2} \omega_{M}^{2}}{\left(\omega_{M}^{2}-\omega^{2}\right)^{2}+\left[\frac{\omega C_{\mathrm{eq}}\left(\omega_{M}\right)}{M}\right]^{2}} \frac{1}{\left(\omega_{n}^{2}-\omega^{2}\right)^{2}}
\end{aligned}
$$

or in non-dimensional form:

$$
e(\xi)=\frac{E(\xi)}{\frac{1}{\omega_{n}^{2}} m V_{0}^{2}}=\frac{\omega^{4} \omega_{n}^{2} \omega_{M}^{2}}{\left(\omega_{M}^{2}-\omega^{2}\right)^{2}+\left[\frac{\omega C_{\mathrm{eq}}(\omega)}{M}\right]^{2}} \frac{1}{\left(\omega_{n}^{2}-\omega^{2}\right)^{2}},
$$

where $\omega_{n}(\xi)$ is replaced for simplicity by $\omega_{n}$. Equation (10) expresses the energy distribution in the attachment at any frequency $\omega$ and depends directly on the frequency distribution $\omega_{n}(\xi)$ and on its derivative $d \omega_{n}(\xi) /\left.d \xi\right|_{\omega_{n}=\omega}$ through the expression for $C_{\text {eq }}$. The total energy over a frequency bandwidth $B$ then becomes

$$
\bar{e}(\xi)=\int_{B} \frac{\omega^{4} \omega_{n}^{2} \omega_{M}^{2}}{\left(\omega_{M}^{2}-\omega^{2}\right)^{2}+\left[\frac{\omega C_{\mathrm{eq}}(\omega)}{M}\right]^{2}} \frac{1}{\left(\omega_{n}^{2}-\omega^{2}\right)^{2}} d \omega .
$$

Invoking Parseval's theorem for the equivalence of frequency- and time-averaging, energy expression in Eq. (11) can also be interpreted as the time average energy of an elemental oscillator located at $\xi$. Requiring $\bar{e}(\xi)$ to be independent of $\xi$, with a constant value $\left(\bar{e}_{0}\right)$ across the attachment, is equivalent to having the energy equally spread over all degrees of freedom $x(\xi)$ :

$$
\int_{B} \frac{\omega^{4} \omega_{n}^{2} \omega_{M}^{2}}{\left(\omega_{M}^{2}-\omega^{2}\right)^{2}+\left[\frac{\omega C_{\mathrm{eq}}(\omega)}{M}\right]^{2}} \frac{1}{\left(\omega_{n}^{2}-\omega^{2}\right)^{2}} d \omega=\bar{e}_{0} .
$$

The functional relationship in Eq. (12) can be solved for $\omega_{n}(\xi)$ numerically within the bandwidth $B$. However, a reduced form of Eq. (12) reveals special properties of the solution $\omega_{n}(\xi)$ around the master frequency. Considering a narrow bandwidth $B$ about $\omega_{M}$ and retaining only the zero-order term of the integrand yields

$$
\frac{B \omega_{M}^{6} \omega_{n}^{2}}{\left.\frac{\omega_{M} C_{\mathrm{eq}}\left(\omega_{M}\right)}{M}\right]^{2}} \frac{1}{\left(\omega_{n}^{2}-\omega_{M}^{2}\right)^{2}} \approx e_{0},
$$

which is valid for $\omega_{n}(\xi) \in B$, i.e., for $\omega_{n}(\xi)$ close to $\omega_{M}$.

Local properties of the frequency distribution can be investigated in terms of the related natural frequency (or modal) density $n$ in the attachment. Considering the number $d N=N d \xi$ of natural frequencies within the interval $d \omega_{n}$, the associated modal density becomes $n\left(\omega_{n}\right)=d N / d \omega_{n}$ $=N d \xi / d \omega_{n}$, which appears explicitly in Eq. (13) through the relationship $\omega_{M} C_{\mathrm{eq}}\left(\omega_{M}\right) / M=\mu(\pi / 4) \omega_{M}^{3} n\left(\omega_{M}\right) / N$. The corresponding expression for $n\left(\omega_{M}\right)$ from Eq. (13) then follows as: 


$$
n\left(\omega_{M}\right)=\frac{4 B N \omega_{n}}{\pi \mu \bar{e}_{0}\left|\omega_{n}^{2}-\omega_{M}^{2}\right|} .
$$

The modal density at the master frequency as given by Eq. (14) is almost singular since $\omega_{n}(\xi) \approx \omega_{M}$. This is exactly the same property of those frequency distributions found in Refs. 9-13 that makes the set of attached resonators a highly effective vibration absorber. When a modal density has such a singularity as that in Eq. (14), expression (10) can be used to show how the master energy vanishes, letting the energy injected into the system almost completely migrate toward the attachment.

Finally, it can be shown that the energy equipartition requirement is the same as the requirement that the modal energies and the time (or frequency) averages of the oscillator energies are equal. The energy of oscillator $j$, expressed as twice that of its kinetic energy, $\Gamma_{j}=m \overline{\dot{x}_{j}^{2}(t)}$, where the bar represents the time average, is also equivalent to its average value in the frequency domain, as shown in Eq. (11). Noting that the modal energy expression $E_{j}$ in Eq. (4) or Eq. (5) is also independent of time, a comparison of $E_{j}$ and $\Gamma_{j}$ can be made using the coordinate transformation in Eq. (3). For the special system under consideration here, where a large number of oscillators are attached to the master in a parallel manner with a total mass small compared to that of the master, modes are localized. For such systems the matrix $\mathbf{U}$ is almost diagonal and the physical and the modal coordinates lead to energies $E_{j}$ and $\Gamma_{j}$ that are substantially similar. As expected, numerical results also show a strong mode localization with an almost diagonal form for the eigenvector matrix $\mathbf{U}$. A proof of the equivalence between $E_{j}$ and $\Gamma_{j}$ for the system described here is presented in Appendix.

\section{DISCUSSION AND CONCLUSIONS}

Earlier studies had shown that vibration energy of a structure can be absorbed nearly irreversibly by a complex attachment that consists of a large number of simple oscillators with the requirement that the attached oscillators possess a particular frequency distribution. These frequency distributions were shown to have a higher modal density around the natural frequency of the master structure. Their distributions were obtained through a variational approach that minimizes the energy associated with the master structure.

The particular form of the frequency distribution deserves a comment on why the frequencies are densely distributed around the frequency to be suppressed and not collocated with it. Selecting the uncoupled frequencies of all the attached oscillators to be the same as that of the natural frequency of the master amounts to constructing a classical tuned absorber that has two degrees of freedom. Considering that the proposed system is conservative, in such a case, an impulse applied to the master would produce a response characterized by two close natural frequencies resulting in a modulation that represents a periodic energy exchange between the master and the satellites that move in unison. However, satellites that are nearly-resonating with the master allow a strong coupling and avoid the simple beat phenomenon described above. The out-of-phase responses are pro- duced by the spread of the resonator frequencies in a small band around the master frequency. The consequence is that soon after they absorb the initial impulse, the oscillators rapidly develop an out-of phase-motion and their total reaction on the master vanishes because of the incoherence of their phases. In a sense, the optimality of the frequency distribution is driven by a compromise between a near-resonant condition and an out-of-phase requirement, leading to the typical frequency form described in this and previous papers.

This paper shows how the frequency distributions obtained previously using a variational approach that minimizes the master energy also result from or are equivalent to an energy equipartition requirement within the system. Finally, an unexpected but significant result for systems as that considered here is that the requirement of energy equipartition among the modal energies is the same as an equipartition among the physical degrees of freedom.

In conclusion, the energy equipartition requirement on the prototypical system described here stores most of the energy in the attachment, leaving $1 / N$ of the total energy in the master, making the attachment an effective energy sink that produces a high damping effect in the master motion.

\section{APPENDIX: EQUIPARTITION AMONG THE MODES AND AMONG THE OSCILLATORS}

Displacement and velocity in expressions for modal energies $E_{j}$ are

$$
\begin{aligned}
& x_{i}(t)=\sum_{j=1}^{N} \frac{U_{i j} \sqrt{2 E_{j}}}{\omega_{j}} \sin \left(\omega_{j} t\right), \\
& \dot{x}_{i}(t)=\sum_{j=1}^{N} U_{i j} \sqrt{2 E_{j}} \cos \left(\omega_{j} t\right) .
\end{aligned}
$$

The energy of the master is given as

$$
E_{N}(t)=\frac{1}{2} M\left(\dot{x}_{N}^{2}(t)+\omega_{M}^{2} x_{N}^{2}(t)\right) .
$$

The time-averaged $E_{N}$ becomes

$$
\left[E_{N}\right]=\lim _{t \rightarrow \infty} \frac{1}{T} \int_{0}^{T} E_{N}(t) d t=\frac{1}{2} M\left(\left[\dot{x}_{N}^{2}\right]+\omega_{M}^{2}\left[x_{N}^{2}\right]\right),
$$

which through Eq. (A1) becomes

$$
\left[E_{N}\right]=E_{\mathrm{tot}} \sum_{j=1}^{N} \frac{U_{N j}^{2}\left(\psi_{j N}\right)^{2}}{2}\left(1+\frac{\omega_{M}^{2}}{\omega_{j}^{2}}\right),
$$

where $E_{\text {tot }}=M V_{0}^{2} / 2$ is the total energy of the system.

The time-average of energy for each resonator of the attachment can be expressed as twice its mean kinetic energy:

$$
\left[E_{i}\right]=m \sum_{j=1}^{N} U_{i j}^{2} E_{j} .
$$

Orthonormality conditions permits expressing $E_{j}$ in terms of $U_{N j}$ :

$$
\mathbf{U}^{T} \mathbf{M U}=\mathbf{I} \rightarrow\left(\mathbf{U}^{-1}\right)^{T}=\mathbf{M U} \rightarrow\left[\mathbf{U}^{-1}\right]_{j N}=M[\mathbf{U}]_{N j},
$$

which when substituted into Eq. (5) yields 


$$
E_{j}=M E_{\mathrm{tot}} U_{N j}^{2}
$$

Thus, the mean time energies can be expressed as

$$
\begin{aligned}
& {\left[E_{N}\right]=\frac{1}{2} \sum_{j=1}^{N} \frac{E_{j}^{2}}{E_{\mathrm{tot}}}\left(1+\frac{\omega_{M}^{2}}{\omega_{j}^{2}}\right),} \\
& {\left[E_{i}\right]=n \sum_{j=1}^{N} U_{i j}^{2} E_{i} .}
\end{aligned}
$$

If energy is equally distributed among all the modes such that $E_{j}=E_{\text {tot }} / N$, for the master-attachment system with an optimal frequency distribution described in Sec. II, the first equation of Eq. (A2) becomes

$$
\left[E_{N}\right]=\frac{1}{2} \frac{E_{\mathrm{tot}}}{N^{2}}\left(N+\sum_{j=1}^{N} \frac{\omega_{M}^{2}}{\omega_{j}^{2}}\right) .
$$

The natural frequencies, as shown in Secs. II and III, have values close to the master frequency, thus allowing an approximation of the summation $\Sigma_{j=1}^{N} \omega_{M}^{2} / \omega_{j}^{2}$ by $N$, yielding

$$
\left[E_{N}\right] \approx \frac{E_{\text {tot }}}{N}
$$

Analogously from the second equation of Eq. (A2), one obtains

$$
\left[E_{i}\right]=\frac{E_{\text {tot }}}{N}\left(m \sum_{j=1}^{N-1} U_{i j}^{2}+\frac{\alpha}{N}\right)
$$

Using the orthonormality conditions, $m \sum_{j=1}^{N-1} U_{i j}^{2}=1-(1 / N)$ :

$$
\left[E_{i}\right] \approx \frac{E_{\mathrm{tot}}}{N} .
$$

Equations (A3) and (A4) show that the optimal frequency distribution produces energy equipartition over the modes as well as over the resonators of the attachment.

${ }^{1}$ A. D. Pierce, V. W. Sparrow, and D. A. Russel, "Fundamental structuralacoustic idealization for structure with fuzzy internals," J. Vibr. Acoust. 117, 339-348 (1995).

${ }^{2}$ M. Strasberg and D. Feit, "Vibration damping of large structures induced by attached small resonant structures," J. Acoust. Soc. Am. 99, 335-344 (1996).

${ }^{3}$ R. L. Weaver, "The effect of an undamped finite degree of freedom 'fuzzy' substructure: Numerical solution and theoretical discussion," J. Acoust. Soc. Am. 101, 3159-3164 (1996).

${ }^{4}$ A. Carcaterra and A. Akay, "Damping device," International Patent No. WO 2006/103291 A1.

${ }^{5} \mathrm{R}$. L. Weaver, "Mean and mean square responses of a prototypical master/ fuzzy system," J. Acoust. Soc. Am. 101, 1441-1449 (1997).

${ }^{6} \mathrm{R}$. L. Weaver, "Equipartition and mean square response in large undamped structures," J. Acoust. Soc. Am. 110, 894-903 (2001).

${ }^{7}$ G. Maidanik, "Induced damping by a nearly continuous distribution of a nearly undamped oscillators: linear analysis," J. Sound Vib. 240, 717-731 (2001).

${ }^{8}$ A. Carcaterra and A. Akay, "Transient energy exchange between a primary structure and a set of oscillators: Return time and apparent damping," J. Acoust. Soc. Am. 115, 683-696 (2004).

${ }^{9}$ I. M. Koç, A. Carcaterra, Z. Xu, and A. Akay, "Energy sinks: Vibration absorption by an optimal set of undamped oscillators," J. Acoust. Soc. Am. 118, 3031-3042 (2005).

${ }^{10}$ A. Akay, Z. Xu, A. Carcaterra, and I. M. Koç, "Experiments on vibration absorption using energy sinks," J. Acoust. Soc. Am. 118, 3043-3049 (2005).

${ }^{11}$ A. Carcaterra, A. Akay, and I. M. Koç, "Nearly irreversible energy trapping by an undamped continuous structure with singularity points in its modal density," J. Acoust. Soc. Am. 119, 2141-2149 (2006).

${ }^{12} \mathrm{~A}$. Carcaterra and A. Akay, "Theoretical foundation of apparent damping and energy irreversible energy exchange in linear conservative dynamical systems," J. Acoust. Soc. Am. 121, 1971-1982 (2007).

${ }^{13}$ A. Carcaterra, A. Akay, and F. Lenti, "Pseudo-damping in undamped plates and shells," J. Acoust. Soc. Am. 122, 804-813 (2007). 